\title{
El currículum de la profesión bibliotecaria; una aproximación
}

\author{
Agustín Gutiérrez Chiñas *
}

Artículo recibido:

22 de agosto de 2012.

Artículo aceptado:

22 de noviembre de 2012.

\section{RESUMEN}

Expone, define y explica el concepto etimológico y operativo de la palabra biblioteca, anotando que la biblioteca es el origen del trabajo bibliotecario que con el paso del tiempo se ha convertido en una profesión con su propio marco conceptual, técnico, teórico y científico. Presenta a grandes rasgos la labor bibliotecaria desde el punto de vista empresarial, mostrando sus tareas administrativas, de organización técnica y de servicio al público. Incluye una propuesta de las acciones principales que hay considerar para elaborar y redactar el currículum de la Licenciatura en Bibliotecología. Explica también algunos valores y principios que sustentan la necesidad de mejorar de manera constante la preparación y formación de los profesionales de la bibliotecología.

* Universidad Autónoma de San Luis Potosí, México. agchinas@uası.m.mx

INVESTIGACIÓN BIBLIOTECOLÓGICA, Vol. 27, Núm. 59, enero/abril, 2013, México, ISSN: 0187-358X. pp. 141-158 
Palabras clave: Profesión bibliotecaria; Educación bibliotecológica; Currículum educativo; Biblioteca; Bibliotecología.

\section{ABSTRACT}

An examination of the curriculum of the professional librarian

Agustín Gutiérrez-Chiñas

The work aims to describe, define and explain the etymological and operational concepts of the term library, noting that the library is the source of the librarian's work, which over time has become a profession with its own conceptual, technical, theoretical and scientific framework. In broad terms, the author presents the work of the librarian from the business standpoint, including administrative tasks, technical organization and service to the public; and offers a proposal for drafting and implementing the course of study for librarianship degree programs. Moreover, the author explains some values and principles that underpin the need to steadily improve the preparation and training of professionals in library and information science.

Keywords: Librarianship; Library education; Curriculum; Library; Library and information science.

\section{INTRODUCCIÓN}

E 1 presente trabajo lleva a cabo una revisión de los elementos que se deben considerar para elaborar un programa educativo, conocido como currículum o currículo, sobre la enseñanza de la profesión bibliotecaria a nivel licenciatura. Para este propósito se procedió a reflexionar sobre las acciones que demanda el servicio bibliotecario, para luego derivar de ellas los elementos que se deben tomar en cuenta para hacer el diseño curricular de la enseñanza de la profesión bibliotecaria y por último presentar una propuesta con los valores que distinguen a la profesión bibliotecaria.

El propósito de este documento es considerar los campos de acción de la Licenciatura en Bibliotecología; es decir, acercarnos al nivel operativo de 
la profesión bibliotecaria con el propósito de rescatar y unificar los criterios directrices con los que nació la educación formal y oficial de la bibliotecología en México, ${ }^{1}$ teniendo en consideración las recomendaciones y propuestas hechas por la Asociación Mexicana de Bibliotecarios, A. C. (AMBAC) ${ }^{2}$ y el Colegio Nacional de Bibliotecarios (CNB), ${ }^{3}$ en sus Mesas Redondas sobre Formación de Recursos Humanos para las Bibliotecas, por un lado; y por el otro, considerar también los cambios que ha venido experimentando la sociedad en los aspectos tecnológico, científico, social y cultural. En este aspecto se puede decir que a pesar de los esfuerzos efectuados para mejorar el contenido de la profesión, el problema de la desvinculación del currículum de la Licenciatura en Bibliotecología con la realidad socioeconómica, técnica, científica y cultural del país continúa latente, debido al quehacer aislado, desarticulado, y a la falta de direccionalidad de conjunto de las instituciones de educación superior (IES) que ofrecen esta licenciatura en México, de acuerdo con lo anotado en los documentos titulados Incompatibilidad curricular de la licenciatura en bibliotecología e información en México y Dispersión curricular en bibliotecología e información en México.

El método y la técnica que se utilizaron fueron las búsquedas bibliográficas con información relacionada para apoyar las anotaciones que se hacen durante el desarrollo de este artículo.

\section{LA PROFESIÓN BIBLIOTECARIA}

El trabajo para obtener, organizar, conservar y difundir toda idea registrada por medio de signos gráficos, iconográficos y fonéticos en un soporte llamado papel, plástico, piel, madera, etc.; en forma de libro, revista, cinta, disco, entre otros formatos; del conocimiento generado y construido por el hombre sobre sí mismo y sobre todos los fenómenos que lo rodean ha sido, es y sigue siendo hasta la fecha tarea o trabajo de la disciplina conocida como bibliotecología.

La historia registra que fueron los griegos clásicos quienes tomaron la iniciativa de poner nombres a los objetos, actividades e instituciones que se distinguían por contar con características comunes en sus funciones. Iniciaron de esta manera el

1 E. Morales Campos, Educación bibliotecológica en México: 1915-1954, p. 5.

2 Mesa Redonda sobre Formación de Recursos Humanos para las Bibliotecas [1], Guanajuato, Gto., 1980, México: AMBAC, 1980. [2] Oaxtepec, Mor., 1981.

3 Mesa Redonda sobre Formación de Recursos Humanos para las Bibliotecas [3], Guanajuato, Gto., 1981, México: CNB, 1982 (Trabajo mecanografiado). [7] México, D. F., 2001.

4 A. Gutiérrez Chiñas, Incompatibilidad curricular de la licenciatura en bibliotecología e información en México, p. 35.

5 A. Gutiérrez Chiñas, "Dispersión curricular en bibliotecología e información en México". 
agrupamiento de lo conocido hasta entonces bajo nombres particulares, que hasta la fecha conservan y utilizan un buen número de sociedades.

Este es el caso del espacio o lugar en el que guardaban sus libros los griegos, que ellos denominaron con dos palabras griegas: biblion que significa libro, y theké que significa armario.

La palabra biblioteca en español se forma con la integración de estas dos palabras, cuyo significado es generalmente entendido como el lugar en el que se guardan o tienen libros. ${ }^{6}$ Este significado ha evolucionado de manera significativa desde el momento en que los griegos concibieron la palabra biblioteca para denominar con ella el lugar para guardar libros. El significado etimológico de biblioteca es un tanto estático en nuestro medio; es decir, generalmente manifiesta la idea de un sitio tranquilo, sin vida, sin acción y sin dinámica, que sólo conserva objetos inanimados que llegaron por sí mismos a este lugar con el fin de esperar su desintegración con el paso del tiempo. Tal vez para la época en que fue acuñada la palabra biblioteca, ésta satisfizo la necesidad de significar el lugar para guardar información registrada en un material concreto, cuyo formato era llamado libro.

A través de los años, aquellos formatos en los que se registra toda idea sobre los conocimientos, las habilidades y las actitudes construidas, adquiridas y desarrolladas por el hombre sobre sí mismo y sobre los fenómenos naturales, artificiales y sociales que lo rodean se multiplicaron con la invención de la imprenta en primer lugar; y en segundo con otros inventos que no sólo aumentaron la cantidad de ideas y conceptos registrados por medio de signos en algún soporte, sino que también incrementaron de manera espectacular la velocidad de producción y distribución de estas ideas por medio de otros formatos además del libro.

El desarrollo abundante y acelerado de la producción y distribución de las ideas registradas en un soporte (información) hizo que la biblioteca, ahora conocida también como unidad de servicios de información documental, pasara de un estado un tanto pasivo, como se concibió en la época de los clásicos griegos, a uno dinámico que poco a poco ha ido cobrando vida por medio de una serie de actividades que realiza o debe realizar actualmente para alcanzar su función social de apoyar a los miembros de una comunidad en su necesidad de tomar una decisión oportuna y acertada, por medio de la información rica, pertinente y adecuada que le oferta en sus servicios.

Pero hacerle llegar información adecuada y acertada de manera oportuna a un miembro de la sociedad implica la ejecución de un conjunto de acciones que no sólo está relacionado con prestarle un documento al individuo 
que lo solicita, que es la idea común que se tiene del servicio de información documental que presta una unidad de información, llámese ésta biblioteca o centro de información. El hecho de prestar un documento que contiene la información que necesita una persona requiere de alguien que haga el préstamo, así como de un local para llevar a cabo los trabajos previos a éste, como son la selección, la adquisición y la organización de la información contenida en cada uno de los documentos que será almacenado y sistematizado (organizado), organización que facilita la localización y recuperación ágil y expedita de cada documento; además de dinero para comprar la materia prima de trabajo, dar mantenimiento, pagar salarios y adquirir equipos, entre otros elementos. Es decir, que prestar un documento informativo de manera oportuna y acertada en los servicios bibliotecarios no se hace sólo por el espíritu contenido en el material documental.

El trabajo para hacer llegar o poner a la disposición de los miembros de una sociedad la información documental que demandan requiere de conocimientos técnicos y teóricos así como de una actitud de servicio por parte de quien lleva a cabo este trabajo. Esto quiere decir que el desarrollo de esta actividad implica conocimientos, competencias, habilidades y actitudes. El conocimiento se relaciona con el entendimiento y la comprensión de la materia prima (los documentos), objeto del trabajo bibliotecario para su selección, adquisición, organización, almacenamiento y recuperación sistematizada; las competencias y habilidades, con la capacidad motriz y técnica para llevar a cabo la ejecución de las acciones anotadas; y las actitudes están relacionadas con la decisión y disposición para servir y apoyar a terceros en la satisfacción de sus necesidades de información documental.

El servicio de información bibliográfica y documental —bibliográfica por el origen etimológico de la palabra biblion y documental por ser el libro un tipo de documento- que brindan las instituciones llamadas bibliotecas y centros de información se apoya en los principios generales de la administración, entendiéndose por ésta el "conjunto sistemático de reglas para lograr la máxima eficiencia en las formas de estructurar un organismo social"7 dentro de un proceso de producción, construcción o servicio. Es decir, que la institución de servicios de información es un organismo social como cualquier otra entidad empresarial creada por la sociedad; que ha decidido llevar a cabo una acción con base en un propósito determinado, generalmente sin fines de lucro. Sin embargo para poder llevar a cabo estas acciones y alcanzar su propósito, la institución también requiere de bienes materiales como un terreno, un edificio, instalaciones, equipo, herramientas y materia prima de 
trabajo, en este caso información registrada, y dinero; además de personas con diferentes niveles de formación y preparación escolar, académica y experiencia; así como un sistema de servicios que demanda de labores de planeación, organización, control, supervisión y evaluación; aspectos que en su conjunto intervienen en el funcionamiento de "una unidad de producción de bienes o servicios para satisfacer un mercado," 8 como cualquier otra unidad empresarial. Se entiende aquí por mercado el lugar público en el que se da la comunicación y el trato entre las personas.

Además de las consideraciones administrativas para el funcionamiento de los servicios informativos documentales que se originan y generan en la biblioteca o centro de información documental, es necesario mencionar y esbozar los procesos a los que se somete la materia prima del trabajo bibliotecario para preparar y elaborar los instrumentos de trabajo que le sirven para brindar sus servicios de información documental, como son el catálogo en sus diferentes modalidades, la bibliografía, la preparación física de los documentos y el marcado o notación que permite la colocación sistemática del material documental en la estantería. La preparación del catálogo y de la bibliografía precisa labores de selección y adquisición del material documental, por un lado; y por otro la organización requiere de la descripción física de cada documento y la clasificación temática de su contenido informativo, datos de descripción y clasificación que se anotan en un registro secundario en elaboración, además de en el mismo documento. El trabajo de selección y adquisición se relaciona con las necesidades de información de la comunidad a la que se pretende llegar y servir y con el mercado editorial de la literatura técnica, científica, cultural y recreativa a nivel local, nacional e internacional. La descripción física demanda conocimientos sobre normas de carácter internacional para construir catálogos, índices, bibliografías y resúmenes; y la clasificación temática requiere del conocimiento y el manejo de sistemas clasificatorios también de uso internacional para el almacenamiento y la recuperación sistematizada de información documental. La preparación física por su parte se lleva a cabo por medio de la inserción de algunos elementos adicionales en cada documento, aun cuando es una actividad modesta y rutinaria también demanda espacio y tiempo para su ejecución; y por último viene el acomodo, la colocación y distribución sistematizadas del material documental en la estantería abierta o cerrada de la unidad informativa, actividades que demandan otra serie de acciones concretas.

El proceso que se le da a la información y a sus envases o soportes en sus diferentes formatos, conocido comúnmente como procesos técnicos, está apoyado 
por el conocimiento que se debe tener sobre el origen, la generación y la naturaleza de la información que se adquiere, organiza, almacena y difunde, así como su envasamiento o registro en algún soporte y su distribución en el mercado editorial. También se debe conocer el idioma en que se encuentra registrada la información, la nacionalidad y movilidad de los generadores de la información (autores), la normatividad que rige la construcción de la estructura de sus registros secundarios, el lenguaje documental y la aplicación de las tecnologías de automatización y telecomunicación en estas labores organizacionales de la información documental.

A la biblioteca, considerada como un organismo social con la intención y finalidad de brindarle información documental a los miembros de una comunidad, le es indispensable que su personal posea conocimientos, competencias, habilidades y, sobre todo, actitudes de servicio para cumplir con su fin último, que es hacer llegar la información documental a quien la necesite; entendiéndose por información "toda idea registrada por medio de signos gráficos, iconográficos y fonéticos en un soporte y formato llamado libro, revista, cinta, disco, etc., del conocimiento generado y construido por

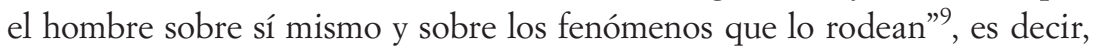
todo mensaje acuñado en un objeto. Para alcanzar esta finalidad la unidad informativa necesita conocer también las necesidades de información de su clientela (usuario) potencial primeramente; y en segundo lugar las estrategias publicitarias y de difusión para alcanzar a esa clientela potencial. Además, el personal debe saber formar y capacitar a sus usuarios en el uso de los recursos informativos; establecer relaciones cordiales con los consumidores de sus servicios; desarrollar, mejorar y actualizar sus servicios de circulación (préstamos), orientación, consultoría, elaboración de bibliografías, estrategias de búsqueda manual y automatizada, entre muchos otros servicios. Para cumplir con este mismo aspecto, el personal debe poseer las competencias, habilidades y actitudes necesarias para establecer contacto y relaciones con otras unidades de información documental e instituciones afines, y para ofrecer y recibir los beneficios de la cooperación con el propósito de prever el comportamiento futuro de los servicios de información, de acuerdo con los cambios que la sociedad vaya experimentando en la técnica, ciencia, educación y cultura.

Con base en el panorama expuesto se puede decir que el trabajo bibliotecario no sólo consiste en guardar y prestar libros sino que va mucho más allá de esta idea estática y tradicional, para convertir a la unidad informativa

9 A. Gutiérrez Chiñas, Manejo de información documental; guia para organizar fuentes de información, p. 12. 
documental en una institución social viva y dinámica que se preocupa por seleccionar, adquirir, organizar, conservar, promover y difundir la información registrada, en igualdad de circunstancias, a todos los miembros de su comunidad para apoyarlos en la satisfacción de sus necesidades y adquirir, corregir, verificar, extender, aplicar y recrear, por medio de la información, su propio conocimiento; también busca propiciar con la información que les brinda el desarrollo de su capacidad imaginativa, inventiva y creativa para reconstruir y construir nuevos conocimientos.

La profesión bibliotecaria se distingue entonces por tres aspectos básicos que debe desarrollar para alcanzar su objetivo de proporcionar información documental a los miembros de la sociedad. Estos aspectos son:

- El administrativo.

- El procesamiento de su materia prima de trabajo.

- La circulación del material informativo, conocido como servicio al público usuario.

Adicionalmente a estos tres aspectos teóricos y prácticos fundamentales de los servicios de información bibliográfica y documental, es necesario incluir los conocimientos, competencias y habilidades profesionales sobre el uso y aplicación de las tecnologías de automatización y telecomunicación en los tres aspectos fundamentales de la profesión bibliotecaria; es decir, el administrativo, el procesamiento de la información (conocido como organización bibliográfica o procesos técnicos), y el de servicio al público.

Para fomentar y propiciar el establecimiento de una actitud comprometida del personal consigo mismo y con la profesión en la que se desempeña, es necesario darle a conocer el origen, desarrollo y los fines de su actividad profesional que le hagan ver, descubrir y reconocer el papel importante que le toca jugar para alcanzar y lograr mejores niveles de bienestar social para su comunidad, de la que él mismo, su familia y amigos forman parte.

Con base en lo ya anotado sobre la profesión bibliotecaria se confirma lo que Shera estableció cuando escribió que "la responsabilidad del bibliotecario es el manejo eficiente y efectivo de la transcripción, del registro de todo lo que la sociedad sabe sobre sí misma y su mundo"10, entendiendo aquí la transcripción como la descripción y organización de la información registrada para propósitos de localización y recuperación. La afirmación de Shera es válida y seguirá siéndolo 
mientras el hombre produzca registros escritos o gráficos con los cuales transmita el conocimiento entre generadores de información y comunicadores [de la misma]. Esa es la esencia y base de nuestra actividad, para ella hemos usado desde tablillas de arcilla hasta supercomputadoras y satélites, pero en el fondo, nuestro papel de transmisores del conocimiento [por medio de la información documental] seguirá siendo el mismo sin importar el instrumento con que lo hagamos. ${ }^{11}$

\section{Currículum}

La palabra currículum desde el punto de vista educativo ha sido abordada y estudiada de diversas maneras y niveles, de tal suerte que "ha rodado por los escenarios más dispares... y ha sido definida en sentidos diversos”. ${ }^{12}$ Sin embargo para poder reflexionar sobre el tema es necesario adoptar un concepto que permita entender su uso y aplicación en el contexto de su estudio y análisis. Por esta razón es recomendable tomar en cuenta que su ubicación epistemológica y teórica le da, o bien un sentido estrecho y fragmentario, o un sentido amplio y totalizador que en ambos casos facilita su entendimiento, sin olvidar que el currículum siempre estará afectado por los cambiantes aspectos históricos, filosóficos, psicológicos, sociales, económicos, políticos y culturales.

El currículum, currículo o programa educativo se crea y genera por la necesidad de organizar y sistematizar en un documento el proceso enseñanzaaprendizaje que se desarrolla en diferentes niveles para preparar y formar a los individuos con el fin de ser útiles a sí mismos, en primer lugar; y segundo, también para los demás miembros de la sociedad. Bajo este considerando educativo que implica aspectos pedagógicos, didácticos y administrativos, el currículum se puede estudiar desde ámbitos formalmente diferenciados como los siguientes:

El punto de vista de su función social en tanto que es el enlace entre la sociedad y la escuela.

Proyecto o plan educativo, pretendido o real, compuesto de diferentes aspectos, experiencias, contenidos, etc., $\mathrm{y}$

Como la expresión formal y material de ese proyecto que debe presentar bajo un formato sus contenidos, orientaciones, secuencias para abordarlos, etc. ${ }^{13}$

11 A. Rodríguez Gallardo, "El papel del nuevo profesional en la bibliotecología".

12 A. Furlán,"Notas y claves para una introducción a la cuestión curricular", p. 257.

13 R. M. Martínez Rider, Evaluación del plan de estudios de la Licenciatura en Biblioteconomía de la Universidad Autónoma de San Luis Potosí, p. 8. 
Visto como un plan, proyecto o programa, el currículum "norma y conduce explícitamente un proceso concreto y determinado de enseñanzaaprendizaje que se desarrolla en una institución educativa". ${ }^{14}$ Por lo mismo el currículum es un "conjunto interrelacionado de conceptos, proposiciones y normas, estructurado en forma anticipada a acciones que se quiere organizar; en otras palabras, es una construcción conceptual destinada a conducir acciones" ${ }^{15}$

El currículum es entonces una articulación de elementos que convergen en la formación y preparación formal y oficial de los educandos de un programa educativo. De estos elementos se distinguen, entre otros, la congruencia interna y externa de la disciplina, la cual permite establecer el perfil del egresado de acuerdo con las demandas sociales; los objetivos curriculares y didácticos; el plan de estudios, los contenidos programáticos de cada materia o asignatura que integra dicho plan, y los planes de cada clase frente al grupo; la evaluación curricular que contemple los métodos, las técnicas y materiales didácticos utilizados; el desempeño docente y el aprovechamiento del alumno, así como también el apoyo administrativo y directivo brindado durante el proceso enseñanza-aprendizaje. Para la construcción de un plan de estudio, como uno de los elementos estructurales del currículum, Díaz Barriga propone los elementos que enseguida se anotan: "a) la construcción de un marco referencial, b) la elaboración de un programa analítico, y c) la interpretación metodológica como un programa guía."16

En consideración a las ideas anotadas sobre el currículum, incluida su definición etimológica y operativa, la cual señala que es una palabra latina que significa conjunto de estudios, puede afirmarse que el

currículum es un documento en el que se establece, claramente definido, el perfil gnoseológico (conocimientos), motriz (competencias y habilidades), y axiológico (actitudes y valores) que debe poseer un individuo al término de la etapa de un proceso en el que participa para su formación y preparación; considerando de manera lógica, cronológica y secuencial los recursos humanos, económicos, materiales, metodológicos y técnicos que se utilicen para llevar a cabo dicho proceso. ${ }^{17}$

Con base en esta idea del currículum se puede decir que la enseñanza y el aprendizaje de una actividad laboral concreta a nivel licenciatura constituyen

14 J. A. Arnaz, La planeación curricular, p. 31.

15 Ibid

16 A. Díaz Barriga, Didáctica y currículum, p. 31.

17 A. Gutiérrez Chiñas, Compatibilidad curricular de la licenciatura en bibliotecología e información en México. 
un fenómeno social que se lleva a cabo entre dos entes: uno singular y otro colectivo o grupal generalmente; basados en una necesidad social que esa profesión pretende satisfacer, derivando de esa actividad laboral concreta las características que el sujeto de la enseñanza y el aprendizaje deberá poseer al término de su paso por el proceso enseñanza-aprendizaje. Durante tal proceso se ponen en juego las competencias, habilidades y actitudes del profesor y del alumno, para alcanzar a concretar en éste las características que definen e identifican a la profesión, y en aquél la mayor responsabilidad para arribar a este fin.

El desarrollo del proceso enseñanza-aprendizaje no se debe dar como un fenómeno espontáneo y arbitrario, aun cuando en un buen número de casos se lleva a cabo de esta manera. Para que alcance sus propósitos este proceso debe ser planificado, organizado y controlado en su implementación y desarrollo, y tanto el trabajo docente como el trabajo de aprendizaje deben sujetarse a estos mencionados tres pasos.

Para estar en condiciones de organizar y sistematizar el proceso enseñanza-aprendizaje de una profesión se debe tomar en cuenta la necesidad social que pretende satisfacer esa profesión, en primer lugar; y en segundo las características particulares y específicas de las actividades concretas indispensables para realizar y satisfacer esa necesidad, la cual se convierte entonces en el factor a considerar para definir, describir y enunciar los conocimientos, competencias, habilidades y actitudes de la profesión que debe enseñar el profesor, y aprehender el alumno.

Con base en la necesidad social detectada, definida y descrita se recomienda proceder con las siguientes acciones:

1. Determinar el nombre de la profesión o licenciatura.

Encontrar la palabra o palabras que sirvan para nombrar una profesión que indique sus cualidades y sus características no es una tarea sencilla, aunque aparentemente así lo parezca. Porque el significado o interpretación que se le puede dar a cada palabra que integre el nombre de la profesión puede influir de manera significativa en su contenido, razón por la cual es recomendable adoptar el nombre de mayor tradición y aceptación entre la comunidad disciplinaria, con el objeto de buscar y conservar la unidad y coherencia de la profesión que permita la compatibilidad de su enseñanza a nivel nacional.

2. Establecer el o los objetivos curriculares.

Para entender el objetivo curricular es necesario partir de la definición operativa del currículum que establece, como ya está anotado, que es un documento (plan) que "norma y conduce, explícitamente, un proceso concreto y determinado de enseñanza-aprendizaje que se 
desarrolla en una institución educativa". ${ }^{18}$ Con esta noción se entiende que el objetivo curricular es el enunciado que describe en forma general los aprendizajes de la profesión que el educando deberá haber obtenido al término de todo el proceso educativo de un nivel determinado. Es decir, el objetivo curricular es la descripción a grandes rasgos de los conocimientos, competencias, habilidades y actitudes que debe poseer el alumno al término de la enseñanza de todos los contenidos del plan de estudio correspondiente.

3. Diseñar el perfil del egresado.

El perfil del egresado es la descripción de cómo será el alumno después de haber transitado por todos los contenidos del plan de estudio. Enuncia de manera más precisa las actividades específicas que será capaz de realizar el alumno, por un lado; y por el otro, explica y clarifica de manera más amplia cada actividad enunciada. A decir de Arnaz, el perfil del egresado es un "retrato hablado, un dibujo a grandes rasgos con las características principales que [el alumno] ha adquirido como resultado de haber transitado por un determinado sistema de enseñanza-aprendizaje". ${ }^{19}$

4. Conformar las áreas académicas.

Con la relación, descripción y explicación de las actividades concretas que deber ser capaz de realizar el alumno, se facilita la conformación de los núcleos básicos conocidos como áreas académicas, de conocimientos, competencias, habilidades y actitudes que necesitará poseer y dominar el alumno para desempeñar su trabajo profesional. Estos núcleos o áreas académicas constituyen los contenidos temáticos que debe enseñar la institución educativa, y los mismos que el alumno por su parte deberá recibir, examinar y registrar en su mente para hacerlos suyos y después aplicarlos en el desempeño de su actividad laboral.

5. Conformar el plan de estudio.

El plan de estudio o retícula es el instrumento curricular que ofrece una visión esquemática de conjunto de las materias o asignaturas de la profesión o licenciatura. Conlleva un sentido de unidad y continuidad con el propósito de darle coherencia a los estudios de la profesión. La conformación del plan de estudio por medio de las asignaturas y la distribución cronológica de éstas en la retícula se debe hacer con base en las áreas académicas que se derivaron del perfil del egresado, que a su vez es producto del objetivo curricular preestablecido. 
6. Elaborar el programa de cada asignatura.

El programa de cada materia es el documento que indica con la mayor precisión posible el objetivo u objetivos de cada materia en particular, las etapas, los métodos, las técnicas y el sistema de evaluación a utilizar para su enseñanza. Este trabajo de planeación de cada materia que integra el plan de estudio es el más laborioso, y se debe hacer en función del tiempo destinado para su impartición.

7. Diseñar el sistema de evaluación.

La medición es el proceso que sirve para conocer y entender la magnitud o calidad de un sistema de producción o de servicio y su producto, que resulta de un fenómeno social basado en un criterio predeterminado. La medición es el medio que el hombre utiliza para asignarle un valor a las cosas y a los fenómenos que lo rodean, a través de una comparación que hace entre un patrón o unidad de medición (criterio predeterminado) y una cantidad, que generalmente representa con números o letras. Esta forma de evaluación, es decir, de asignar valores, sirve para controlar todo el proceso curricular de la enseñanza-aprendizaje de una licenciatura o profesión, desde su planeación, implementación, desarrollo y conclusión para atraer la atención del alumno hacia lo más importante de su preparación, para retroalimentar el mecanismo de aprendizaje, para detectar el bajo aprendizaje o aprendizaje insuficiente, para conocer los resultados del método, de la técnica y de los recursos utilizados y también para evaluar la actuación del profesor así como para asignar calificaciones que validen y legitimen la formación escolar y académica alcanzada por el alumno.

De las siete acciones anotadas, el inciso 5 (elaborar el programa de cada asignatura) es el que está mayormente relacionado con el proceso enseñanzaaprendizaje propiamente dicho, porque mediante la elaboración del programa de enseñanza de cada asignatura se establecen los objetivos didácticos que el profesor y el alumno deben alcanzar de manera conjunta. En esta acción se enuncian de manera separada las funciones que el profesor y el alumno llevarán a cabo de manera simultánea en el mismo espacio y tiempo durante el proceso. El programa de cada materia también señala los métodos, las técnicas y los recursos que utilizará el profesor, así como también las actividades que deberá realizar el alumno para lograr su aprendizaje. Asimismo, el programa debe señalar el espacio y tiempo en los que se llevará a cabo el proceso enseñanza-aprendizaje de la materia en cuestión.

Además de la elaboración del programa de cada materia, el proceso enseñanza-aprendizaje también exige una labor de planeación de cada sesión de trabajo 
docente frente al grupo. Esta labor de planeación incluye la redacción de los objetivos específicos u operativos que se deben exponer y alcanzar en cada sesión de trabajo conjunto entre el profesor y el educando, considerando la hora como la unidad de medición que se utiliza para controlar del tiempo disponible para la enseñanza de cada asignatura. El plan de trabajo (plan de clase) frente al grupo y con el grupo sólo incluye los objetivos operativos, porque en el programa de cada asignatura ya están considerados los objetivos terminales o generales y particulares o intermedios de la asignatura, los cuales se concretizan en la medida en que se logran los objetivos operativos o específicos precisamente. Y en la medida que se realicen los objetivos generales de cada materia, se irán alcanzando también los objetivos curriculares de la profesión correspondiente.

Por último, el desarrollo en vivo y a todo color del proceso enseñanzaaprendizaje, es decir, del acto docente en acción, también debe llevar a cabo el dominio del manejo de grupo por parte del profesor. Este dominio significa que el docente debe poseer un conocimiento amplio del tema y de la disciplina que enseña, mediante el manejo de un lenguaje comprensible, rico y variado; una actitud y un comportamiento positivos y comprometidos con su asignatura y la profesión, y un uso apropiado de sus gestos y movimientos corporales. Es decir, que el profesor debe ser un actor que vive, siente y escenifica lo que enseña.

\section{CONSIDERACiOnes Finales}

¿Cuáles son los valores de la profesión bibliotecaria, qué es lo que hace importante a esta licenciatura y por qué vale la pena estudiarla?

Para responder a estas interrogantes es necesario reflexionar sobre la materia prima de trabajo del servicio bibliotecario. Esta materia prima se llama información, ¿qué es la información y para qué sirve?

Desde el punto de vista etimológico, J. Zeman establece que el término información se deriva de la palabra latina informare, que significa "poner en forma, dar forma o un aspecto, formar... representar, presentar, crear una idea o una noción". ${ }^{20}$ Por su parte, Iguíniz señala que el vocablo en cuestión es la traducción de la palabra también latina informatione, cuyo significado es "diseño, imagen e idea". ${ }^{21}$

Efectivamente la información es toda idea registrada, como ya se anotó, por medio de símbolos gráficos, iconográficos y fonéticos en un soporte cuyo 
formato puede ser un libro, una revista, una cinta, un disco, etc., del conocimiento generado y construido por el hombre sobre sí mismo y sobre los demás fenómenos que lo rodean, como los naturales, artificiales y sociales.

Además de la definición etimológica existen otras de carácter operativo que establecen que la información es "todo suceso capaz de transmitir conocimiento entre un emisor y un receptor", 22 otra más dice que es el "dato, antecedente e indicio sobre el origen, desarrollo y los efectos de un fenómeno natural o social", ${ }^{23}$ y otra más reza que es "todo aquello que reduce la incertidumbre", ${ }^{24}$ entre otras definiciones.

Gracias a esta materia prima llamada información, el servicio bibliotecario se encuentra en una posición estratégica para participar como apoyo en el desarrollo de la sociedad, fomentando en ella con la información documental que le proporciona una capacidad de pronóstico de gran exactitud, para llevar a cabo una toma de decisión oportuna y acertada y evitar de esta manera el azar en sus acciones, con lo que disminuye en un alto porcentaje la duplicidad de esfuerzos, movimientos y recursos.

Desde el punto de vista académico, es decir desde el enfoque relacionado con las actividades de la docencia, el estudio, la investigación e innovación, la información sirve:

1. Para adquirir un conocimiento. Es decir, que gracias a la información se puede hacer propia una o más ideas, y combatir de esta manera la ignorancia.

2. Para corregir un conocimiento. Esto es, que permite enmendar, reparar y subsanar una idea equivocada, con el propósito de mejorarla.

3. Para verificar un conocimiento. Gracias a la información se puede revisar y probar la veracidad de una idea o concepto del que se duda y se tiene incertidumbre.

4. Para extender un conocimiento. Significa que la información puede ampliar y aumentar la cantidad de ideas y nociones que se poseen sobre un tema en particular, enriqueciendo la cantidad, diversidad y variedad de los subtemas.

5. Para aplicar un conocimiento en el proceso de construcción, fabricación y elaboración de un producto, así como también en el diseño y la oferta de un servicio.

6. Para recrear o disfrutar un conocimiento por medio de la lectura de un documento que contiene información de carácter histórico, novelístico y poético, entre otros géneros literarios.

22 Teoría de la Información, en Enciclopedia Británica, p. 176.

23 A. Gutiérrez Chiñas, "El catedrático como promotor del uso y aprovechamiento de la información".

24 G. S. Foibisoff y E. P. Donald, "Information and information needs". 
7. Para generar o crear un nuevo conocimiento, que es la máxima y maravillosa capacidad que tiene el hombre para imaginar, inventar y participar de manera innovadora en el mejoramiento de su desarrollo personal y colectivo o social.

Además de los valores implícitos en la materia prima (información documental) del trabajo bibliotecario, la profesión promueve el uso y aprovechamiento de la información registrada en soportes. Esta labor de promoción la lleva a cabo por medio de su trabajo de selección, adquisición, organización, almacenamiento, y de la búsqueda y recuperación sistematizada de información documental que oferta a sus usuarios. De esta manera la profesión le aporta un servicio valioso a la sociedad, porque le ahorra dinero, tiempo y esfuerzo a sus miembros cuando éstos andan en la búsqueda, localización, recuperación y adquisición del nutriente que satisface sus necesidades intelectuales y espirituales. Nutriente que les sirve para desarrollar su ingenio y capacidad creativa, inventiva y recreativa. En los tiempos actuales el valor del trabajo bibliotecario calificado, es decir, preparado y formado a nivel licenciatura, aumenta de manera considerable por la ayuda que les ofrece a los miembros de la sociedad para lidiar con la identificación de sus necesidades de información documental, y también para encontrar los medios para satisfacer éstas, en un mundo en donde la redundancia informativa apabulla al individuo, no en un mar, sino en un océano de documentos, dada la explosión bibliográfica y documental que hoy en día se vive.

Por último se anota que la profesión bibliotecaria es también valiosa porque en ella se encuentran los principios y fundamentos técnicos, metodológicos y teóricos que sustentan y soportan el desarrollo filosófico, conceptual, teórico, técnico y científico de la disciplina llamada bibliotecología; sin olvidar la cobertura a nivel internacional que debe tomar en consideración la propuesta de unificación y homologación de criterios que hace la Declaración de Bolonia por lo que toca a la denominación de la profesión en sus diferentes niveles de formación, y la adopción de un número de ciclos o niveles de educación superior (licenciatura, maestría y doctorado) con miras a establecer un sistema internacional de créditos que propicie y facilite la promoción y el fomento de la movilidad de estudiantes, profesores, investigadores y personal administrativo y de servicios. ${ }^{25}$ 


\section{Bibliografía CONSULTADA}

Arnaz, J. A., La planeación curricular, México: Nuevomar, 1984, 74 p.

Díaz Barriga, A., Didáctica y currículum, 2 ed., México: Nuevomar, 1984, 150 p.

, "La disolución del concepto de currículum", en Perspectivas Docentes, (6):35-40, México, 1991.

Díaz Barriga Arceo, F., "Aproximaciones metodológicas al diseño curricular: hacia una propuesta integral", en Tecnología y Comunicación Educativas, (21):19-39, México, 1993.

Dicionario de la Lengua Española, 19 ed., Madrid: Real Academia Española, 1970.

Foibisoff, G.S. y E.P. Donald,"Information and information needs", en Information Reports and Bibliographies, 5(5):2, EUA, 1973.

Furlán, A." Notas y claves para una introducción a la cuestión curricular", en Foro de Análisis del Currículum de la Licenciatura en Pedagogía. Memorias, México: 1985.

Gutiérrez Chiñas, A., Compatibilidad curricular de la Licenciatura en bibliotecología e información en México, tesis de Doctorado en Pedagogía, México: UNAM, 2001.

"Dispersión curricular en bibliotecología e información en México”, en Ramos Fandiño, G. P.; Mata Puente, A.; Oliva Cruz, E. (eds.), Aportaciones a la bibliotecología y a las ciencias de la información, San Luis Potosí, SLP: UASLP/ECI, 2011, pp. 103-137, ISBN: 978-607-7856-53-5.

, "El catedrático como promotor del uso y aprovechamiento de la información”, en Conciencia Universitaria, UASLP, 4(16), 1990.

Incompatibilidad curricular de la licenciatura en bibliotecología e información en México, San Luis Potosí, SLP: UASLP/COPOCYT, 2009, 224 p.

, "La indisciplina en la enseñanza de la disciplina bibliotecológica en México", en Ciencias de la Información, 36(1):39-65, Cuba, abril 2005.

Manejo de información documental; guia para organizar fuentes de información, 2 ed., México: Trillas, 2012.

Iguíniz, J. B., Léxico bibliográfico, México: UNAM-Biblioteca Nacional de México, 1959, 307 p.

Martínez Rider, R. M., Evaluación del plan de estudios de la Licenciatura en Biblioteconomía de la Universidad Autónoma de San Luis Potosí, tesis de Maestría en Educación; Campo Formación Docente, San Luis Potosí, SLP: UPN-Unidad 24-A, 1996, 95 p.

Mesa Redonda sobre Formación de Recursos Humanos para las Bibliotecas [1], Guanajuato, Gto., 1980, México: AMBAC, 1980 [2] Oaxtepec, Mor., 1981.

[3], Guanajuato, Gto., 1981, México: CNB, 1982 (Trabajo mecanografiado), [7] México, D.F., 2001. 
Morales Campos, E., Educación bibliotecológica en México: 1915-1954, México: UNAM/CUIB, 1988, 112 p.

Proceso de Bolonia, http.//es.wikipedia.org./wiki/Proceso_de_Bolonia [consultado: 7-nov-2012].

Reyes Ponce, A., Administración de empresas; teoría y práctica, México: Limusa, 1984, v. 1.

Rodríguez Gallardo, A., "El papel del nuevo profesional en la bibliotecología”, en Información, 8(34), México, 1998, pp. 7-11.

Sacristán, G., El currículum; una reflexión sobre la práctica, 2 ed., Madrid: Morata, 1989, 150 p.

Shera, J. H., Los fundamentos de la educación bibliotecológica, México: UNAM-CUIB, 1990.

Teoría de la Información, en Enciclopedia Británica, Barcelona: Enciclopedia Británica, 1991-1992, v. 8, p. 176.

Zeman, J., "Significación filosófica de la idea de información”, en El concepto de información contemporánea, $3^{\mathrm{a}}$ ed., México: Siglo XXI, 1975, (Coloquios de Royaumont). 